\title{
Weighted Tradeoff between Effective Capacity and Energy Efficiency
}

\author{
Wenjuan Yu, Leila Musavian and Qiang Ni \\ School of Computing and Communications, InfoLab21, \\ Lancaster University, Lancaster, UK, \\ Email: \{w.yu1, 1.musavian, q.ni\}@lancaster.ac.uk
}

\begin{abstract}
This paper proposes a new power allocation technique to jointly optimize link-layer energy efficiency (EE) and effective capacity (EC) of a Rayleigh flat-fading channel with delay-outage probability constraints. Specifically, EE is formulated as the ratio of $\mathrm{EC}$ to the sum of transmission power and rate-independent circuit power consumption. A multi-objective optimization problem (MOP) to jointly maximize EE and EC is then formulated. By introducing importance weight into the MOP, we can flexibly change the priority level of EE and EC, and convert the MOP into a single-objective optimization problem (SOP) which can be solved using fractional programming. At first, for a given importance weight and a target delay-outage probability, the optimum average transmission power level to maximize the SOP is found. Then, the optimal power allocation strategy is derived based on the obtained average input power level. Simulation results confirm the analytical derivations and further show the effects of circuit power, importance weight, and transmission power constraint limit on the achievable tradeoff performance.
\end{abstract}

\section{INTRODUCTION}

During the last decade, climate change has emerged as a global challenge and many governments, academics and industries are now increasingly unified in a call to action [1]. It is reported that information and communications technology (ICT) industry is estimated to contribute between $2 \%$ to $3 \%$ of global greenhouse gas emissions [2], a share which is quickly raising. Besides, although silicon technology is exponentially progressing, the power consumption of the processor is also increasing by $150 \%$ every two years [3]. In contrast, the improvement in battery technology is much more sluggish, about $10 \%$ increase every two years [3], which leads to a rapidly increasing gap between the demand for energy and the battery capacity offered. Therefore, to meet the challenges raised by the high demands of wireless traffic and energy consumption, green communication has become an urgent need. Energy efficiency (EE), in b/J/Hz, and spectral efficiency (SE), in $\mathrm{b} / \mathrm{s} / \mathrm{Hz}$, are considered as two key performance indicators for green wireless communication systems. Unfortunately, it is known that $\mathrm{EE}$ and $\mathrm{SE}$ are inconsistent and conflict with each other.

To tackle this problem, many studies on the EE-SE tradeoff have been carried out [4]-[10]. In particular, the EESE tradeoff problem was formulated as a constrained optimization model, for interference-limited wireless networks in [4], downlink orthogonal frequency division multiple access (OFDMA) networks in [5], and cooperative cognitive radio networks in [6]. In the aforementioned studies, EE was fixed as the objective function and a constraint on achievable rate

This work was supported in part by the China Scholarship Council, UK EPSRC under grant number EP/K011693/1 and the EU FP7 under grant number PIRSES-GA-2013-610524. was imposed. However, according to the dynamic property of surrounding circumstances, various application types, and different users' preferences, the corresponding objective function might need to change. Therefore, continuously adjustable objective function is indispensable, but it is not provided in [4]-[6].

The EE-maximization problem was converted into an MOP in [7] and the weighted sum method was introduced to convert the MOP into an SOP, subject to constraints on overall transmit power of each remote access unit, bit error rate, and proportional data rates for mobile stations. The MOP approach in an OFDMA cellular network was provided in [8] which introduced the normalization factor and transformed the MOP into an SOP using weighted sum method. Later, considering tradeoff between $\mathrm{EE}$ and SE, the power loading problem for orthogonal frequency division multiplexing (OFDM) with imperfect channel estimation was investigated in [9]. Specifically, instead of maximizing both EE and SE, the inverse of EE and inverse of SE were minimized to make shannon capacity as the common denominator [9]. The weighted sum method was also used in [9] to build a tractable tradeoff function.

In the aforementioned tradeoff papers [4]-[9], shannon limit was given as the system throughput, which is suitable for systems with no link-layer QoS requirement. However, for enabling multimedia communication systems, delay QoS requirement has been an essential factor [11]. In such systems, effective capacity (EC) can be used to specify the maximum constant arrival rate with a target delay-outage probability requirement [11]. Therefore, the link-layer EE is defined as the ratio of EC to the total expenditure power. However, EE and EC could conflict with each other. In more details, the EE curve as a function of transmit power has a bell shape where the location of its maximum depends on the circuit power [12]. On the other hand, EC is a monotonically increasing function of transmission power [11]. Therefore, depending on the operational transmit power, EE and EC may conflict, and hence, how to balance the two metrics deserves elaborate study.

With the theory of link-layer EC, the relationship between link-layer EE and EC under delay constraint was exploited in [10]. Firstly, singal-to-noise ratio (SNR) was expressed in terms of EC, using a curve fitting method. Then EE, as a function of SNR, is defined as a function of EC. Therefore, the relationship curve between EE and EC could be provided. However, the mathematical formulation of tradeoff between $\mathrm{EE}$ and EC, as well as the close-form power allocation strategy was not provided.

In this paper, considering a system with delay-outage probability requirement, a new link-layer EE-EC tradeoff 
formulation is proposed and then, solved using fractional programming. Before starting to integrate the joint EE-EC maximization problem, we transform the two objectives into two normalized unitless functions, to get rid of their different measurements and orders of magnitude. Further, instead of maximizing link-layer $\mathrm{EE}$ and $\mathrm{EC}$, we minimize the inverse of $\mathrm{EE}$ and the inverse of $\mathrm{EC}$ to make $\mathrm{EC}$ as the common denominator. We then introduce the importance weight into the formulation, which could be used to adjust the priority of EE and EC according to surrounding circumstances, user's preference and system's QoS requirement. By transforming the MOP into an SOP, we show that the tradeoff function is quasi-concave in transmission power, and hence, a global maximum could be achieved through fractional programming [13]. For a point-to-point Rayleigh flat-fading channel with delay constraints, we present numerical results to illustrate the effects of the importance weight, circuit power and transmission power constraint limit on the tradeoff problem.

\section{SySTEM MODEL}

We consider a point-to-point wireless communication system over a flat-fading channel. Similar to what is considered in [14], firstly, the upper-layer packets are divided into frames at the data-link layer. At the physical layer, the frames stored at a first-in-first-out (FIFO) buffer are split into bit streams. Adaptive coding and power allocation strategy are applied at the transmitter [15], using the channel-state information (CSI) fed back from the receiver, and the QoS constraint. Then, the bit streams are read out of the FIFO buffer and transmitted through the wireless fading channel.

We assume that the wireless channel is block fading, i.e., the channel gain is invariant during each fading-block, but independently changes from one fading-block to another. The length of each fading-block, denoted by $T_{\mathrm{f}}$, is assumed to be an integer multiple of the symbol duration $T_{\mathrm{s}}$. We also assume that the ideal Nyquist transmission symbol rate is satisfied, which means that the symbol duration $T_{\mathrm{s}}$ equals to the inverse of the system bandwidth, $T_{\mathrm{s}}=\frac{1}{B}$. During each fading-block, the service rate process, $\left\{R[t], t=1,2, \ldots, T_{\mathrm{f}}\right\}$, using adaptive transmission is considered to be stationary and ergodic [14]. Therefore, the block index $t$ could be omitted for simplicity. The instantaneous service rate, in $\mathrm{b} / \mathrm{s} / \mathrm{Hz}$, at the $t^{t h}$ fading-block is given as

$$
R[t]=\log _{2}\left(1+P_{t}[t] \cdot \frac{\gamma[t]}{P_{\mathcal{L}} \sigma_{\mathrm{n}}^{2}}\right) \quad(\mathrm{b} / \mathrm{s} / \mathrm{Hz}),
$$

where $P_{t}[t]$ is the transmission power, $P_{\mathcal{L}}$ denotes the distance-based path-loss, $\sigma_{\mathrm{n}}^{2}$ indicates the noise power and $\gamma[t]$ presents the channel power gain of the considered unitvariance Rayleigh block fading channel with the probability density function (PDF) $f_{\gamma}(\gamma)=\mathrm{e}^{-\gamma}[16]$.

\section{A. Effective Capacity}

Based on the large deviation theory, for a dynamic queueing system with stationary ergodic arrival and service processes [17], the queue length process $Q(t)$ converges in distribution to a steady-state queue length $Q(\infty)$ such that

$$
-\lim _{x \rightarrow \infty} \frac{\ln (\operatorname{Pr}\{Q(\infty) \geq x\})}{x}=\theta,
$$

where $\operatorname{Pr}\{a \geq b\}$ shows the probability that $a \geq b$ holds. (2) implies that the probability of the queue length exceeding a certain threshold $x$ decays exponentially fast as $x$ increases [15].

Now, assuming that the Gartner-Ellis theorem [18, Pages 34-36] is satisfied, EC of an independent and identically distributed (i.i.d) block fading channel can be expressed as [11]

$$
\mathrm{EC}=-\frac{1}{\theta T_{\mathrm{f}} B} \ln \left(\mathbb{E}\left[e^{-\theta B T_{\mathrm{f}} R[t]}\right]\right) \quad(\mathrm{b} / \mathrm{s} / \mathrm{Hz}),
$$

where $\mathbb{E}[\cdot]$ indicates the expectation operator. Note that in (3), the parameter $\theta(\theta>0)$ indicates the exponential decay rate of the QoS violation probability. A slower decay rate could be represented by a smaller $\theta$, which indicates that the system can tolerate a looser QoS guarantee, while a more stringent QoS requirement will be indicated by a larger $\theta$.

Now, the delay-outage probability, which is defined as the probability that the delay exceeds a maximum delay-bound $D_{\max }$, can be estimated by [11]

$$
P_{\text {delay }}^{\text {out }}=\operatorname{Pr}\left\{\text { Delay } \geq D_{\max }\right\} \approx \varepsilon \mathrm{e}^{-\theta \mu D_{\max }},
$$

where $D_{\max }$ is in units of a symbol period $\left(T_{\mathrm{s}}=1 / B\right)$. Hence, in order to meet a target delay-bound violation probability

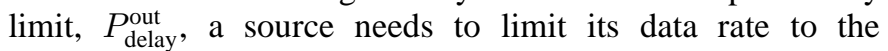
maximum of $\mu$, where $\mu$ is the solution of $\mu=\mathrm{EC}$, given in (3).

\section{B. Link-layer Energy Efficiency}

We formulate the link-layer EE for delay-limited system as the ratio of EC to the sum of the circuit power, $P_{\mathrm{c}}$, and the transmission power scaled by the power amplifier efficiency $\epsilon$. Therefore, EE can be expressed as

$$
\mathrm{EE}=\frac{\mathrm{EC}}{P_{\mathrm{c}}+\frac{1}{\epsilon} \mathbb{E}\left[P_{\mathrm{t}}[t]\right]} \quad 0 \leq \epsilon \leq 1 .
$$

\section{Optimal Power Allocation}

In this section, we formulate the EE-EC tradeoff as an MOP and provide the optimal power allocation strategy under average input power constraint. Since EE and EC have different measurements and orders of magnitude, we normalize them with $\mathrm{EE}_{\text {norm }}$ and $\mathrm{EC}_{\text {norm }}$, respectively. To be specific, if $P_{\mathrm{EE}}^{*}$ denotes the optimal average power level at which $\mathrm{EE}$ is maximized, then $\mathrm{EE}_{\mathrm{norm}}=\left.\mathrm{EE}\right|_{\mathbb{E}\left[P_{\mathrm{t}}[t]\right]=P_{\mathrm{EE}}^{*}}$ and $\mathrm{EC}_{\mathrm{norm}}=$ $\left.\mathrm{EC}\right|_{\mathbb{E}\left[P_{\mathrm{t}}[t]\right]=P_{\mathrm{EE}}^{*}} ^{*}$

Here, instead of jointly maximizing $\frac{\mathrm{EE}}{\mathrm{EE}_{\text {norm }}}$ and $\frac{\mathrm{EC}}{\mathrm{EC}_{\mathrm{norm}}}$, we minimize the inverse of the two conflicting objectives to make EC as the common denominator. The MOP, hence, can be formulated as

$$
\begin{aligned}
& \min \frac{\mathrm{EE}_{\mathrm{norm}}}{\mathrm{EE}} \text { and } \min \frac{\mathrm{EC}_{\mathrm{norm}}}{\mathrm{EC}} \\
& \text { subject to: } \mathbb{E}\left[P_{\mathrm{t}}[t]\right] \leq P_{\max },
\end{aligned}
$$

where $P_{\max }$ is the average transmission power limit. By utilizing (4), we combine the two objectives in (5a) using an importance weight, which could be adjusted to indicate the priority of different objectives. Therefore, the MOP is transformed into an SOP with input power constraint, which 
yields

$$
\min w_{1} \frac{\mathrm{EE}_{\text {norm }}\left(P_{\mathrm{c}}+\frac{1}{\epsilon} \mathbb{E}\left[P_{\mathrm{t}}[t]\right]\right)}{\mathrm{EC}}+\left(1-w_{1}\right) \frac{\mathrm{EC}_{\text {norm }}}{\mathrm{EC}}
$$

subject to: $\mathbb{E}\left[P_{\mathrm{t}}[t]\right] \leq P_{\max }$,

where $w_{1} \in[0,1]$ is the importance weight. In more details, $w_{1}$ and $\left(1-w_{1}\right)$ represent the importance of the link-layer EE and EC, respectively. When $w_{1}=0$, the tradeoff problem is transformed into an EC-maximization problem, while when $w_{1}=1$, the MOP is simplified into an EE-maximization problem. In fact, the importance of EE gradually grows as $w_{1}$ increases from 0 to 1 .

Then, since the transmission power is a function of the channel power gain $\gamma$, the instantaneous transmission power $P_{\mathrm{t}}[t]$ in (6a)-(6b) could be written as $P_{\mathrm{t}}(\gamma)$. Finally, the EEEC tradeoff problem can be mathematically expressed as

$$
\begin{aligned}
& \min _{P_{\mathrm{r}}(\gamma) \geq 0} w_{1} \frac{\mathrm{EE}_{\text {norm }} K_{\ell}\left(P_{\mathrm{c}_{\mathrm{r}}}+\frac{1}{\epsilon} \mathbb{E}_{\gamma}\left[P_{\mathrm{r}}(\gamma)\right]\right)}{-\frac{1}{\theta T_{\mathrm{f}} B} \ln \left(\mathbb{E}_{\gamma}\left[\left(1+P_{\mathrm{r}}(\gamma) \gamma\right)^{-\alpha(\theta)}\right]\right)} \\
&+\left(1-w_{1}\right) \frac{1}{-\frac{1}{\theta T_{\mathrm{f}} B} \ln \left(\mathbb{E}_{\gamma}\left[\left(1+P_{\mathrm{r}}(\gamma) \gamma\right)^{-\alpha(\theta)}\right]\right)}
\end{aligned}
$$

subject to: $\mathbb{E}_{\gamma}\left[P_{\mathrm{t}}(\gamma)\right] \leq P_{\max }$,

where $\mathbb{E}_{\gamma}[\cdot]$ indicates the expectation over the PDF of $\gamma$, $P_{\mathrm{r}}(\gamma)=\frac{P_{\mathrm{t}}(\gamma)}{K_{\ell}}, P_{\mathrm{c}_{\mathrm{r}}}=\frac{P_{\mathrm{c}}}{K_{\ell}}, \alpha(\theta)=\frac{\theta T_{\mathrm{f}} B}{\ln 2}, K_{\ell}=P_{\mathcal{L}} \sigma_{\mathrm{n}}^{2}$. Setting $\mathrm{EE}_{\text {norm }}^{\prime}=\mathrm{EE}_{\text {norm }} K_{\ell}, K_{\ell}$ could be canceled to scale the normalized system performance with respect to path-loss factor. Since $-\frac{1}{\theta T_{\mathrm{f}} B}$ is a negative constant, the minimization problem (7a) can be converted into a maximization problem. Then, it could be converted back into a minimization problem by inverting the objective function, yielding

$$
\min _{P_{\mathrm{r}}(\gamma) \geq 0} \frac{\ln \left(\mathbb{E}_{\gamma}\left[\left(1+P_{\mathrm{r}}(\gamma) \gamma\right)^{-\alpha(\theta)}\right]\right)}{w_{1} \mathrm{EE}_{\text {norm }}^{\prime}\left(P_{\mathrm{c}_{\mathrm{r}}}+\frac{1}{\epsilon} \mathbb{E}_{\gamma}\left[P_{\mathrm{r}}(\gamma)\right]\right)+\left(1-w_{1}\right) \mathrm{EC}_{\text {norm }}}
$$

subject to: $\mathbb{E}_{\gamma}\left[P_{\mathrm{r}}(\gamma)\right] \leq \frac{P_{\max }}{K_{\ell}}$.

\section{A. Optimum Power Allocation With No Input Power Con- straint}

Firstly, the unconstrained SOP is tackled, paving the way for the optimum power allocation strategy of the SOP with input average power constraint. The objective function in (8a) involves the ratio of two functions of $P_{\mathrm{r}}(\gamma)$, and it is not concave [13]. However, the EC function has been proved to be concave in the transmission power, and in turn, in $P_{\mathrm{r}}(\gamma)$ [14]. Therefore, the numerator in (8a) is convex [19]. On the other hand, the denominator of the objective function is affine in $P_{\mathrm{r}}(\gamma)$, therefore, the tradeoff problem is quasiconcave and could be solved through fractional programming [13].

Now, by using the variable transformation $t=\left(w_{1} \operatorname{EE}_{\text {norm }}^{\prime}\left(P_{\mathrm{c}_{\mathrm{r}}}+\frac{1}{\epsilon} \mathbb{E}_{\gamma}\left[P_{\mathrm{r}}(\gamma)\right]\right)+\left(1-w_{1}\right) \mathrm{EC}_{\text {norm }}\right)^{-1}$, the minimization problem (8a)-( $8 \mathrm{~b})$ could be converted into

$$
\min _{P_{\mathrm{r}}(\gamma) \geq 0} \quad t \ln \left(\mathbb{E}_{\gamma}\left[\left(1+P_{\mathrm{r}}(\gamma) \gamma\right)^{-\alpha(\theta)}\right]\right)
$$

$$
\begin{gathered}
\text { subject to: } \quad t\left(w_{1} \mathrm{EE}_{\text {norm }}^{\prime}\left(P_{\mathrm{c}_{\mathrm{r}}}+\frac{1}{\epsilon} \mathbb{E}_{\gamma}\left[P_{\mathrm{r}}(\gamma)\right]\right)\right. \\
\left.+\left(1-w_{1}\right) \mathrm{EC}_{\text {norm }}\right)=1 .
\end{gathered}
$$

The objective function in $(9 \mathrm{a})$ is convex in $\left(P_{\mathrm{r}}(\gamma), t\right)$ with an affine constraint [13]. Therefore, the Karush-Kuhn-Tucker (KKT) conditions are both sufficient and necessary for the optimal solution [19]. If $\lambda \in \mathbb{R}$ is the Lagrange multiplier, then the Lagrangian is

$$
\begin{aligned}
& \mathcal{L}\left(P_{\mathrm{r}}(\gamma), t, \lambda\right)= \\
& t \ln \left(\mathbb{E}_{\gamma}\left[\left(1+P_{\mathrm{r}}(\gamma) \gamma\right)^{-\alpha(\theta)}\right]\right)+\lambda\left(t \left(w_{1} \mathrm{EE}_{\text {norm }}^{\prime}\right.\right. \\
& \left.\left.\left(P_{\mathrm{c}_{\mathrm{r}}}+\frac{1}{\epsilon} \mathbb{E}_{\gamma}\left[P_{\mathrm{r}}(\gamma)\right]\right)+\left(1-w_{1}\right) \mathrm{EC}_{\text {norm }}\right)-1\right) .
\end{aligned}
$$

At the optimal power allocation, we have

$$
\begin{aligned}
& \frac{\partial \mathcal{L}\left(P_{\mathrm{r}}(\gamma), t, \lambda\right)}{\partial P_{\mathrm{r}}(\gamma)}=0, \\
& \frac{\partial \mathcal{L}\left(P_{\mathrm{r}}(\gamma), t, \lambda\right)}{\partial t}=0,
\end{aligned}
$$

which can be found as

$$
\begin{aligned}
& \alpha(\theta) \gamma\left(1+P_{\mathrm{r}}(\gamma) \gamma\right)^{-\alpha(\theta)-1} \\
& =\frac{\lambda w_{1} \mathrm{EE}_{\text {norm }}^{\prime}}{\epsilon} \mathbb{E}_{\gamma}\left[\left(1+P_{\mathrm{r}}(\gamma) \gamma\right)^{-\alpha(\theta)}\right] \\
& \ln \left(\mathbb{E}_{\gamma}\left[\left(1+P_{\mathrm{r}}(\gamma) \gamma\right)^{-\alpha(\theta)}\right]\right) \\
& +\lambda\left(w_{1} \mathrm{EE}_{\text {norm }}^{\prime}\left(P_{\mathrm{c}_{\mathrm{r}}}+\frac{1}{\epsilon} \mathbb{E}_{\gamma}\left[P_{\mathrm{r}}(\gamma)\right]\right)\right. \\
& \left.+\left(1-w_{1}\right) \mathrm{EC}_{\text {norm }}\right)=0 .
\end{aligned}
$$

From (12a), the optimum power distribution scheme can be found as

$$
P_{\mathrm{r}}(\gamma)=\left[\frac{\alpha(\theta)^{\frac{1}{1+\alpha(\theta)}}}{\left(w_{1} \nu\right)^{\frac{1}{1+\alpha(\theta)}} \gamma^{\frac{\alpha(\theta)}{1+\alpha(\theta)}}}-\frac{1}{\gamma}\right]^{+},
$$

where $[x]^{+}=\max \{0, x\}$ and $\nu=$ $\frac{\lambda \mathrm{EE}_{\text {norm }}^{\prime}}{\epsilon} \mathbb{E}_{\gamma}\left[\left(1+P_{\mathrm{r}}(\gamma) \gamma\right)^{-\alpha(\theta)}\right]$ is referred to as the scaled-Lagrangian-multiplier. The optimal value for $\nu$ (referred to as $\nu^{*}$ ) can be found by substituting the power allocation (13) into (12b), yielding

$$
\begin{aligned}
& \mathrm{EE}_{\text {norm }}^{\prime} \mathbb{E}_{\gamma}\left[\left(1+\left[\frac{(\gamma \alpha(\theta))^{\frac{1}{1+\alpha(\theta)}}}{\left(w_{1} \nu^{*}\right)^{\frac{1}{1+\alpha(\theta)}}}-1\right]^{+}\right)^{-\alpha(\theta)}\right] \\
& \times \ln \left(\mathbb{E}_{\gamma}\left[\left(1+\left[\frac{(\gamma \alpha(\theta))^{\frac{1}{1+\alpha(\theta)}}}{\left(w_{1} \nu^{*}\right)^{\frac{1}{1+\alpha(\theta)}}}-1\right]^{+}\right)^{-\alpha(\theta)}\right]\right) \\
& +\epsilon \nu^{*}\left(w _ { 1 } \operatorname { E E } _ { \text { norm } } ^ { \prime } \left(P_{\mathrm{c}_{\mathrm{r}}}+\frac{1}{\epsilon} \mathbb{E}_{\gamma}\left[\frac{\alpha(\theta)^{\frac{1}{1+\alpha(\theta)}}}{\left(w_{1} \nu^{*}\right)^{\frac{1}{1+\alpha(\theta)}} \gamma^{\frac{\alpha(\theta)}{1+\alpha(\theta)}}}\right.\right.\right. \\
& \left.\left.\left.-\frac{1}{\gamma}\right]^{+}\right)+\left(1-w_{1}\right) \mathrm{EC}_{\text {norm }}\right)=0 .
\end{aligned}
$$

For a Rayleigh fading channel, the expectations in (14) can be calculated by (15a) and (15b), wherein $\Gamma(a, x)=\int_{x}^{\infty} t^{a-1} \mathrm{e}^{-t} d t$ is the upper incomplete gamma function and $\mathrm{E}_{1}(x)=\int_{x}^{\infty} \frac{\mathrm{e}^{-t}}{t} d t,|\operatorname{Arg}(x)|<\pi$ indicates the 


$$
\begin{aligned}
\mathbb{E}_{\gamma}\left[P_{\mathrm{r}}(\gamma)\right] & =\left(\frac{\alpha(\theta)}{w_{1} \nu^{*}}\right)^{\frac{1}{1+\alpha(\theta)}} \Gamma\left(\frac{1}{1+\alpha(\theta)}, \frac{w_{1} \nu^{*}}{\alpha(\theta)}\right)-\mathrm{E}_{1}\left(\frac{w_{1} \nu^{*}}{\alpha(\theta)}\right) \\
\mathbb{E}_{\gamma}\left[\left(1+P_{\mathrm{r}}(\gamma) \gamma\right)^{-\alpha(\theta)}\right] & =\left(\frac{w_{1} \nu^{*}}{\alpha(\theta)}\right)^{\frac{\alpha(\theta)}{1+\alpha(\theta)}}(1+\alpha(\theta))\left[-\left(\frac{w_{1} \nu^{*}}{\alpha(\theta)}\right)^{\frac{1}{1+\alpha(\theta)}} \mathrm{e}^{-\frac{w_{1} \nu^{*}}{\alpha(\theta)}}\right. \\
& \left.+\Gamma\left(\frac{2+\alpha(\theta)}{1+\alpha(\theta)}, \frac{w_{1} \nu^{*}}{\alpha(\theta)}\right)\right]+1-\Gamma\left(1, \frac{w_{1} \nu^{*}}{\alpha(\theta)}\right),
\end{aligned}
$$

exponential integral [20]. Based on the derivations in (13)(15b), the power allocation strategy could be summarized as follows.

Remark 1: The optimum power allocation technique for MOP of the link-layer EE and EC, at a target delay-outage probability, includes two steps.

In Step $1, \nu^{*}$ could be found by substituting (15a) and (15b) into (14). The operating input power level $P_{\text {toff }}^{*}$, corresponding to the maximum achievable tradeoff performance, can then be found by inserting $\nu^{*}$ into (15a), namely

$$
P_{\text {toff }}^{*}=K_{\ell} \times\left.\mathbb{E}_{\gamma}\left[P_{\mathrm{r}}(\gamma)\right]\right|_{\nu=\nu^{*}}
$$

In Step 2, we optimally distribute the transmit power based on $P_{\text {toff }}^{*}$. Since $P_{\text {toff }}^{*}$ is a unique optimum value, the denominator of the EE-EC tradeoff function (8a) is fixed and equal to $w_{1} \mathrm{EE}_{\text {norm }}^{\prime}\left(P_{\mathrm{c}_{\mathrm{r}}}+\frac{1}{\epsilon K_{\ell}} P_{\text {toff }}^{*}\right)+\left(1-w_{1}\right) \mathrm{EC}_{\text {norm }}$. Hence, the formulated SOP in (8a) simplifies to

$$
\begin{aligned}
& \max _{P_{\mathrm{r}}(\gamma) \geq 0}-\frac{1}{\theta T_{\mathrm{f}} B} \ln \left(\mathbb{E}_{\gamma}\left[\left(1+P_{\mathrm{r}}(\gamma) \gamma\right)^{-\alpha(\theta)}\right]\right) \\
& \text { subject to: } \quad \mathbb{E}_{\gamma}\left[P_{\mathrm{r}}(\gamma)\right] \leq \frac{P_{\text {toff }}^{*}}{K_{\ell}} .
\end{aligned}
$$

Finally, the optimum power distribution is given in (13).

\section{B. Optimal Power Allocation under Average Input Power Constraint}

Here, the EE-EC tradeoff problem under an average input power constraint, given in (6a)-(6b), is considered. Using the results of Section III-A, the problem (8a)-(8b) simplifies to

$$
\begin{array}{ll}
\max _{P_{\mathrm{r}}(\gamma) \geq 0} & -\frac{1}{\theta T_{\mathrm{f}} B} \ln \left(\mathbb{E}_{\gamma}\left[\left(1+P_{\mathrm{r}}(\gamma) \gamma\right)^{-\alpha(\theta)}\right]\right) \\
\text { subject to: } & \mathbb{E}_{\gamma}\left[P_{\mathrm{r}}(\gamma)\right] \leq \frac{P_{\text {toff }}^{*}}{K_{\ell}} \\
& \mathbb{E}_{\gamma}\left[P_{\mathrm{r}}(\gamma)\right] \leq \frac{P_{\max }}{K_{\ell}} .
\end{array}
$$

Hence, the power-constrained EE-EC tradeoff problem reduces into a power-constrained EC-maximization problem, and the average input power limit becomes $\min \left(P_{\text {toff }}^{*}, P_{\max }\right)$.

\section{NuMERICAL RESUlTS}

In this section, we numerically investigate the impact of circuit power, transmission power constraint and importance weight on EE-EC tradeoff problem for a Rayleigh block-fading channel with delay-outage probability constraints.

Firstly, Fig. 1 plots the EE, in $b / J / H z$, versus importance weight, $w_{1}$, for various circuit power values. The figure reveals that the link-layer EE proportionally increases with $w_{1}$. This happens because the increase of $w_{1}$

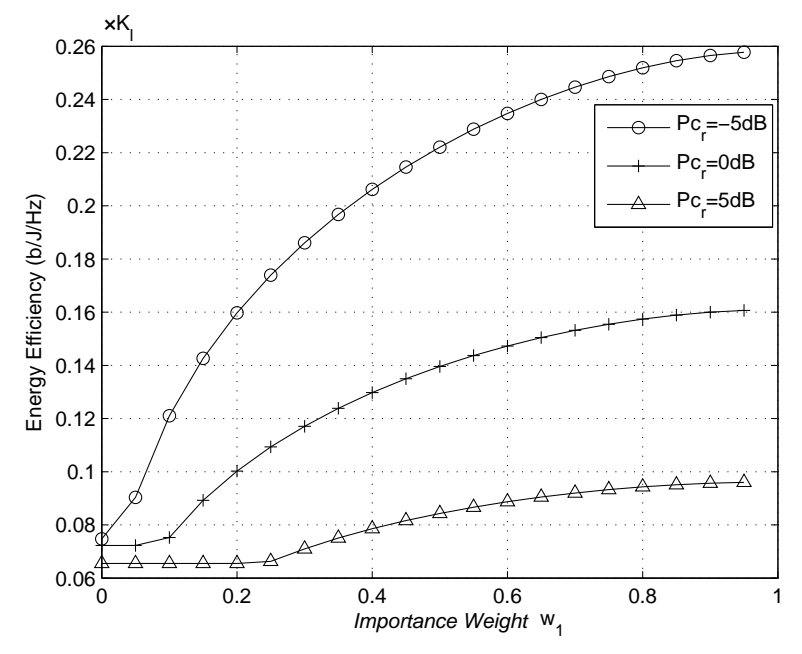

Fig. 1: Energy efficiency versus importance weight, for various scaled circuit power values.

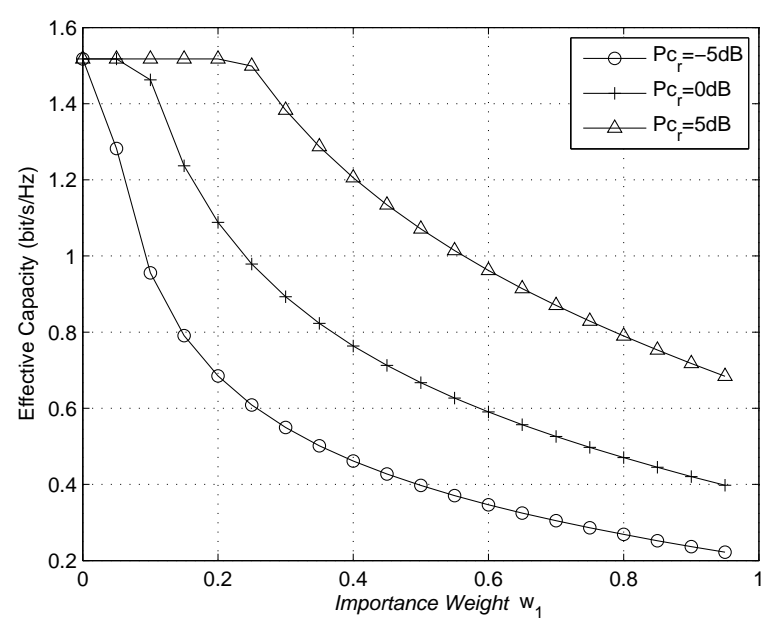

Fig. 2: Effective capacity versus importance weight, for various scaled circuit power values.

raises the importance of $\frac{E_{\text {norm }}}{E E}$. Moreover, Fig. 1 shows that with fixed $w_{1}$, when $P_{\mathrm{c}_{\mathrm{r}}}$ increases from $-5 \mathrm{~dB}$ to $5 \mathrm{~dB}$, EE decreases. We can show that, the average input power limit $P_{\text {toff }}^{*}$ increases monotonically with the circuit power, $P_{\mathrm{c}}$. Since EE varies inversely with $P_{\mathrm{c}}+P_{\text {toff }}^{*}$, EE decreases with $P_{\mathrm{C}_{\mathrm{r}}}$.

EC versus importance weight for various $P_{\mathrm{c}_{\mathrm{r}}}$ is illustrated in Fig. 2. Particularly, for a given $P_{\mathrm{c}_{\mathrm{r}}}$, when $w_{1}$ increases, 


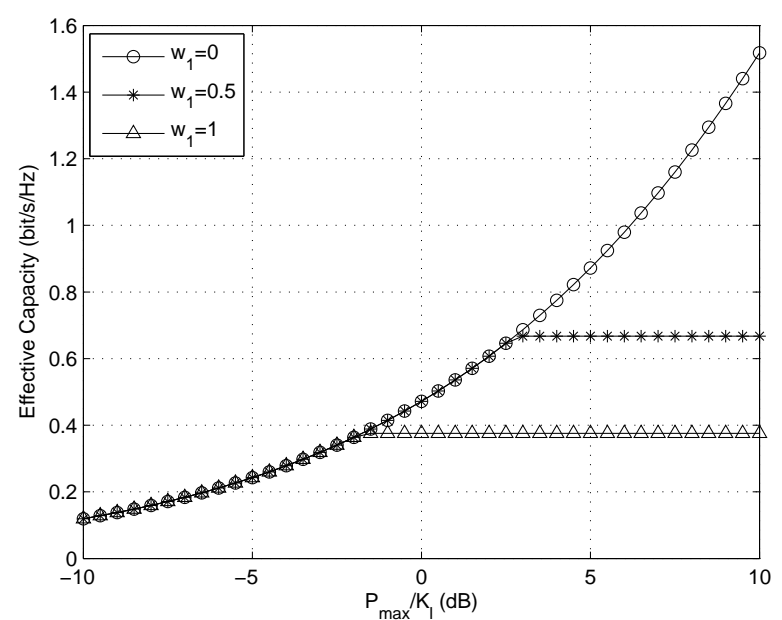

Fig. 3: Effective capacity versus scaled average input power limit, for various importance weights.

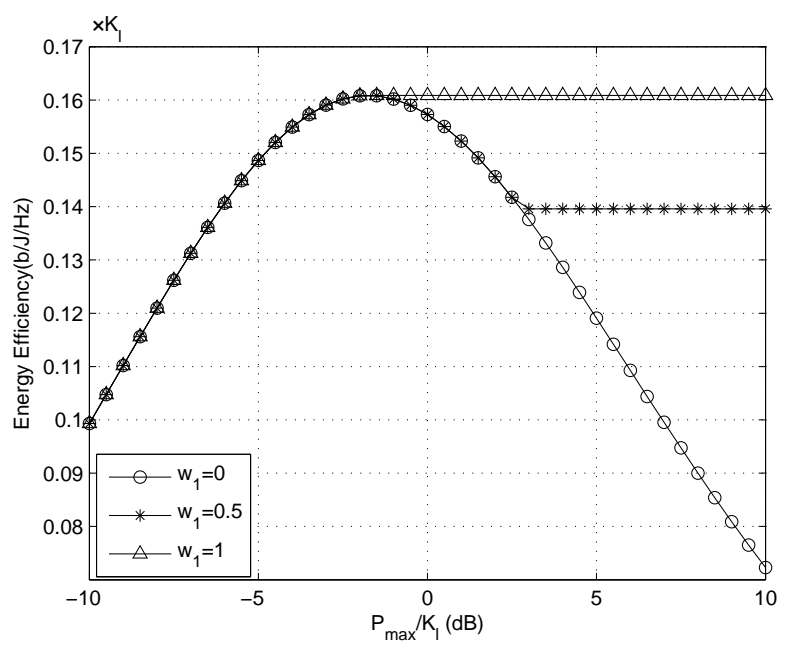

Fig. 4: Energy efficiency versus scaled average input power limit, for various importance weights.

EC gradually decreases. This indicates that $\frac{\mathrm{EC}_{\text {norm }}}{\mathrm{EC}}$ is less important with bigger $w_{1}$. In addition, in both Fig. 1 and Fig. 2, there is a flat region wherein $\mathrm{EE}$ and $\mathrm{EC}$ remain constant with increasing $w_{1}$, that is when $w_{1} \in\left[\begin{array}{ll}0, & 0.2\end{array}\right]$, for $P_{\mathrm{c}_{\mathrm{r}}}=5 \mathrm{~dB}$. It happens because, when $P_{\mathrm{c}_{\mathrm{r}}}$ is large and $w_{1}$ is small, $\frac{\mathrm{EC}_{\text {norm }}}{\mathrm{EC}}$ dominates the tradeoff function. As a result, the tradeoff system prefers to maximize EC. Hence, $\left.\mathrm{EC}\right|_{w_{1} \leq 0.2}=\left.\mathrm{EC}\right|_{w_{1}=0},\left.\mathrm{EE}\right|_{w_{1} \leq 0.2}=\left.\mathrm{EE}\right|_{w_{1}=0}$, where $w_{1}=0$ represents the EC-maximization problem. When $w_{1}>0.2$, the system starts to maximize $\frac{\mathrm{EE}_{\mathrm{norm}}}{\mathrm{EE}}$ as well, so the curve will not stay flat.

We further plot the results for EC versus $P_{\max }$, and EE versus $P_{\max }$, with $P_{\mathrm{c}_{\mathrm{r}}}=0 \mathrm{~dB}$ in Fig. 3 and Fig. 4, respectively. In particular, Fig. 3 shows that the tradeoff system with $w_{1} \neq 0$ achieves the same EC as an EC-maximization system, i.e., $w_{1}=0$, until it reaches a break-point, after which EC flattens. This happens because, after the breakpoint $P_{\text {toff }}^{*}$, increasing power does not benefit the tradeoff performance. As shown in Section III-B, for any $P_{\text {toff }}^{*} \leq P_{\max }$, the operational input power

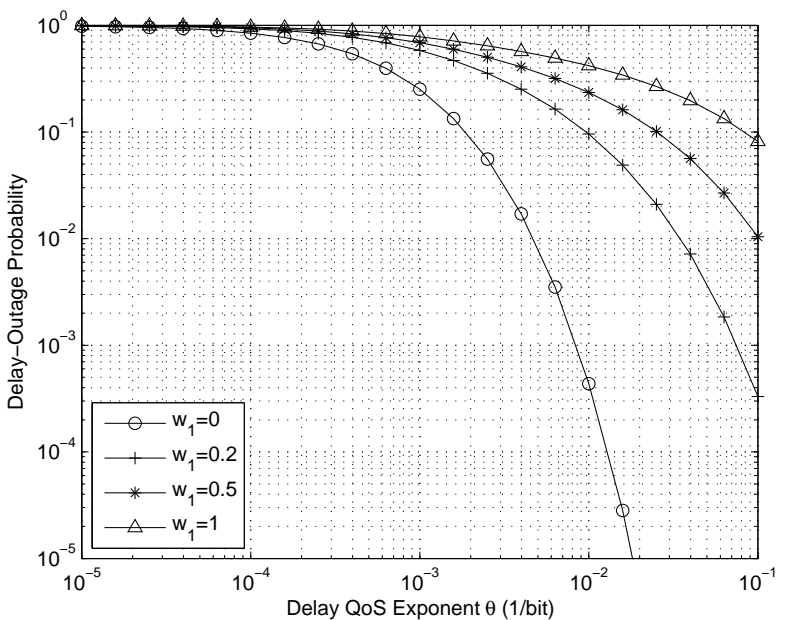

Fig. 5: Delay-outage probability versus delay QoS exponent, for various importance weights.

constraint will be remained at $P_{\text {toff }}^{*}$. Therefore, the tradeoff problem does not consume all the available power, but rather operates at a fixed power level of $P_{\text {toff }}^{*}$, and in turn, achieves a fixed EC, when $P_{\text {toff }}^{*} \leq P_{\max }$.

In the tradeoff system with $w_{1}=0.5$ plotted in Fig. 4, EE increases with $P_{\max }$ to a maximum value, and then decreases until a break-point, after which it remains constant. In other words, with the increase of $P_{\max }$, EE increases to the maximum value $\mathrm{EE}_{\text {norm }}$, then decreases until the breakpoint $P_{\text {toff }}^{*}$ is reached. In EE-maximization situation, i.e., $w_{1}=1$, the achievable EE increases with the increase of $P_{\max }$ until a break-point at $P_{\mathrm{EE}}^{*}$. This is due to the fact that the system operates at the global optimal power level $P_{\mathrm{EE}}^{*}$ for any $P_{\mathrm{EE}}^{*} \leq P_{\max }$. On the other hand, in EC-maximization situation, $w_{1}=0$, EE decreases after it reaches its maximum. It is because the EC-optimized power allocation strategy consumes the whole available input power, resulting in continuously growing EC, and simultaneously losing EE.

Finally, the delay-outage probability, $P_{\text {delay, }}^{\text {out }}$ versus delay QoS exponent, $\theta$, for various importance weights, with a maximum tolerable delay threshold $D_{\max }=500, P_{\mathrm{c}_{\mathrm{r}}}=$ $-10 \mathrm{~dB}$ is plotted in Fig. 5. This figure indicates that for loose delay-constrained systems, e.g., $\theta=10^{-5}$, different importance weights will not affect the achievable $P_{\text {delay }}^{\text {out }}$ On the other hand, when $\theta$ increases, e.g., $\theta \geq 10^{-3}$, smaller $w_{1}$ provides smaller delay-outage probability. This happens because smaller $w_{1}$ indicates that the system prefers ECmaximization approach, hence, the system achieves higher EC with smaller $w_{1}$. Therefore, the probability that the data is remained in the FIFO buffer is decreased. As a result, the probability that the delay of a symbol exceeds a maximum delay-bound $D_{\max }$ decreases.

\section{CONCLUSIONS}

We formulated and solved link-layer EE-EC tradeoff problem, for a point-to-point Rayleigh flat-fading channel under delay-outage probability constraint, using the weighted sum method and fractional programming. In order to make the two objectives comparable, we normalized $\mathrm{EE}$ and $\mathrm{EC}$ with $\mathrm{EE}_{\text {norm }}$ and $\mathrm{EC}_{\text {norm }}$, respectively. We then minimized the inverse of the two conflicting objectives and transformed the MOP into 
a scalar criteria optimization problem with the introduction of an importance weight. Finally, the optimal power allocation strategy for the power-constrained tradeoff problem on determined weight was given. Numerical results showed that when circuit power is large, the tradeoff performance is more favorable towards maximizing EC.

\section{REFERENCES}

[1] C. Despins, F. Labeau, T. L. Ngoc, R. Labelle, M. Cheriet, C. Thibeault, F. Gagnon, A. Leon-Garcia, O. Cherkaoui, B. S. Arnaud, J. McNeill, Y. Lemieux, and M. Lemay, "Leveraging green communications for carbon emission reductions: Techniques, testbeds, and emerging carbon footprint standards," IEEE Communications Magazine, vol. 49, no. 8, pp. 101-109, Aug. 2011.

[2] Smart2020, "Enabling the low-carbon economy in the information age," The Climate Group, London, U.K., Tech. Rep., 2008.

[3] K. Lahiri, A. Raghunathan, S. Dey, and D. Panigrahi, "Battery-driven system design: A new frontier in low power design," in IEEE Int. Conf. on VISI Design, Bangalore, India, Jan. 2002, pp. 261-267.

[4] Y. Li, M. Sheng, C. Yang, and X. Wang, "Energy efficiency and spectral efficiency tradeoff in interference-limited wireless networks," IEEE Communications Letters, vol. 17, no. 10, pp. 1924-1927, Oct. 2013.

[5] C. Xiong, G. Y. Li, S. Zhang, Y. Chen, and S. Xu, "Energy- and spectral-efficiency tradeoff in downlink OFDMA networks," IEEE Trans. Wireless Commun., vol. 10, no. 11, pp. 3874-3886, Nov. 2011.

[6] X. Chen and S. Ouyang, "Energy- and spectral-efficiency trade-off in OFDMA-based cooperative cognitive radio networks," International Journal of Distributed Sensor Networks, Feb. 2014.

[7] C. He, B. Sheng, P. Zhu, X. You, and G. Y. Li, "Energy- and spectralefficiency tradeoff for distributed antenna systems with proportional fairness," IEEE Journal on Selected areas in Communications, vol. 31, no. 5, pp. 894-902, May 2013.

[8] J. Tang, D. K. C. So, E. Alsusa, and K. A. Hamdi, "Resource efficiency: A new paradigm on energy efficiency and spectral efficiency tradeoff," IEEE Trans. on Wireless Communications, vol. 13, no. 8, pp. 1536-1276, Aug. 2014.

[9] O. Amin, E. Bedeer, M. H. Ahmed, and O. A. Dobre, "Energy efficiency and spectral efficiency trade-off for OFDM systems with imperfect channel estimation," in IEEE Int. Conf. Commun. (ICC 2014), Sydney, Australia, Jun. 2014, pp. 3553-3558.

[10] X. Chen, R. Q. Hu, G. Wu, and Q. C. Li, "Tradeoff between energy efficiency and spectral efficiency in a delay constrained wireless system," Wireless Communications and Mobile Computing, Mar. 2014.

[11] D. Wu and R. Negi, "Effective capacity: A wireless link model for support of quality-of-service," IEEE Trans. Wireless Commun., vol. 2, no. 4, pp. 630-643, Jul. 2003.

[12] G. Li, Z. Xu, C. Xiong, C. Yang, S. Zhang, Y. Chen, and S. Xu, "Energyefficient wireless communications: tutorial, survey, and open issues," IEEE Wireless Commun., vol. 18, no. 6, pp. 28-35, Dec. 2011.

[13] S. Schaible, "Minimization of ratios: Techinical notes," Journal of Optimization Theory and Applications, vol. 19, no. 2, pp. 347-352, Jun. 1976.

[14] L. Musavian and T. Le-Ngoc, "Energy-efficient power allocation over nakagmi-m fading channels under delay-outage constraints," IEEE Trans. Wireless Commun., vol. 13, no. 8, pp. 4081-4091, Aug. 2014.

[15] J. Tang and X. Zhang, "Quality-of-service driven power and rate adaptation over wireless links," IEEE Trans. Wireless Commun., vol. 6, no. 8, pp. 3058 - 3068, Aug. 2007.

[16] M. K. Simon and M.-S. Alouini, Digital Communication over Fading Channels: A Unified Approach to Performance Analysis. John Wiley and Sons Inc., 2000.

[17] C. S. Chang, "Stability, queue length, and delay of deterministic and stochastic queueing networks," IEEE Trans. Automatic Control, vol. 39, no. 5, pp. 913-931, May 1994.

[18] J. A. Bucklew, Introduction to Rare Event Simulation. Springer-Verlag New York Inc., 2004.

[19] S. Boyd and L. Vandenberghe, Convex Optimization. Cambridge University Press, 2004.

[20] M. Abramowitz and I. A. Stegun, Handbook of mathematical functions. New York: Dover, 1965. 